\title{
Caractérisation physico-chimique et source de la minéralisation des eaux souterraines des départements de Daloa et Zoukougbeu, Côte d'Ivoire
}

\author{
Oi Adjiri ADJIRI ${ }^{1 *}$, Bintou KONE ${ }^{3}$, Natchia AKA ${ }^{2}$, Ibrahim DJABAKATE ${ }^{1}$ et Brou DIBI ${ }^{1}$ \\ ${ }^{1}$ Laboratoire des Sciences et Technologies de l'Environnement (LSTE), UFR Environnement, Université Jean \\ Lorougnon GUEDE, BP 150 Daloa, Côte d'Ivoire. \\ ${ }^{2}$ Laboratoire de physique et géologie marine, Centre de Recherches Océanologiques d'Abidjan (CRO), BP \\ V18 Abidjan, Côte d'Ivoire. \\ ${ }^{3}$ Département de Mines et Réservoirs (DMR), UFR Sciences Géologiques et Minières, Université de Man, BP \\ 20 Man, Côte d'Ivoire. \\ "Auteur correspondant : E-mail : oiadjiri@gmail.com; Tel : (+225) 224332 38/0760 2906
}

\section{RÉSUMÉ}

Cette étude vise à faire la caractérisation hydrogéochimique et à identifier les facteurs de minéralisation des eaux souterraines. Pour y parvenir, 14 échantillons de sols et 17 échantillons d'eaux de sources et puits ont été prélevés dans 12 localités différentes. Ils ont été conditionnés respectivement dans des sachets alimentaires et dans des bouteilles en PET de $1 \mathrm{~L}$. Ces dernières ont été conditionnées dans des glacières à $4 \pm 2{ }^{\circ} \mathrm{C}$. Auparavant, les paramètres température, $\mathrm{pH}$, conductivité électrique, TDS et turbidité, des eaux ont été mesurés in situ. Les valeurs moyennes sont respectivement : $27,63{ }^{\circ} \mathrm{C} ; 4,81 ; 84,67 \mu \mathrm{S} / \mathrm{cm} ; 38,45 \mathrm{mg} / \mathrm{L}$ et 27,22 UTN. Les analyses physico-chimiques réalisées sur les sols, indiquent un pH moyen de 4,84 et des concentrations moyennes de $\mathrm{NO}_{3}{ }^{-}, \mathrm{NO}_{2}{ }^{-}$et $\mathrm{NH}_{4}{ }^{+}$respectives de 93,$19 ; 0,20$ et $0,54 \mathrm{ppm}$. Le dosage chimique des eaux a donné des teneurs moyennes de $\mathrm{HCO}_{3}{ }^{-}, \mathrm{Cl}^{-}, \mathrm{NO}_{3}{ }^{-}$et $\mathrm{SO}_{4}{ }^{2-}$ respectives de 10,$73 ; 8,42 ; 8,05$ et 5,31 $\mathrm{mg} / \mathrm{L}$ et de $\mathrm{Ca}^{2+}, \mathrm{Mg}^{2+}, \mathrm{K}^{+}$et $\mathrm{Na}^{+}$respectives de 2,$21 ; 1,03 ; 1,84$ et $5,18 \mathrm{mg} / \mathrm{L}$. Les simulations PHREEQC et diagramme indiquent un système fermé à l'atmosphère, des eaux sous-saturées par rapport aux carbonates et évaporites et donc, agressives et de temps de séjour court.

(C) 2019 International Formulae Group. All rights reserved.

Mots clés: Daloa, Zoukougbeu, paramètres hydrogéochimiques, diagramme 5.0, minéralisation, PHREEQC.

\section{Physico-chemical characterization and source of groundwater mineralization of the Daloa and Zoukougbeu departments, Ivory Coast}

\begin{abstract}
This study aims to perform hydrogeochemical characterization and to identify groundwater mineralization factors. To achieve this, 14 soil samples and 17 spring and well water samples were collected from 12 different locations. They were packaged in food bags and in PET bottles of 1L respectively. The water samples were packaged in coolers at $4 \pm 2{ }^{\circ} \mathrm{C}$. Before, temperature, $\mathrm{pH}$, electrical conductivity, TDS and turbidity of water were measured in situ. The average values were $27.63{ }^{\circ} \mathrm{C} ; 4.81 ; 84.67 \mu \mathrm{S} / \mathrm{cm} ; 38.45 \mathrm{mg} / \mathrm{L}$ and 27.22 NTU respectively. Physico-chemical analyzes of soils showed mean values of $\mathrm{pH}, \mathrm{NO}_{3}{ }^{-}, \mathrm{NO}_{2}{ }^{-}$and
\end{abstract}


$\mathrm{NH}_{4}{ }^{+}$were 4.84, $93.19 \mathrm{ppm}, 0.20 \mathrm{ppm}$ and $0.54 \mathrm{ppm}$ respectively. The chemical analyses of water showed that the concentrations of $\mathrm{HCO}_{3}{ }^{-}, \mathrm{Cl}^{-}, \mathrm{NO}_{3}{ }^{-}$and $\mathrm{SO}_{4}{ }^{2-}$ were $10.73 ; 8.42 ; 8.05$ and $5.31 \mathrm{mg} / \mathrm{L}$ respectively and those of $\mathrm{Ca}^{2+}, \mathrm{Mg}^{2+}, \mathrm{K}^{+}$and $\mathrm{Na}^{+}$were $2.21 ; 1.03 ; 1.84$ and $5.18 \mathrm{mg} / \mathrm{L}$ respectively. The PHREEQC and Diagram simulations indicated a closed system to the atmosphere and undersaturated water with respect to carbonates and evaporites. These characteristics indicate aggressive waters and short residence time.

(C) 2019 International Formulae Group. All rights reserved.

Keywords: Daloa, Zoukougbeu, hydrogeochemical parameters, diagramme 5.0, mineralization, PHREEQC.

\section{INTRODUCTION}

Les eaux souterraines sont d'une importance capitale dans la plupart des régions du monde pour l'approvisionnement en eau potable. Elles peuvent aussi être captées par l'homme grâce à des puits ou forages (De Marsily et al., 2017). La résurgence de ces eaux génère les eaux de source. Lorsque ces dernières ne sont pas contaminées, elles ont les mêmes caractéristiques que les eaux souterraines. D'autre part, en dehors des facteurs climatiques et anthropogènes, la composition chimique d'une eau souterraine est tributaire des caractéristiques des roches encaissantes et des substances réactives qu'elle peut rencontrer lors de son infiltration (Mohan et al., 2000 ; Rajmohan et al., 2000 ; Boubakar, 2011) ou de son émergence. En conséquence, ces trois facteurs sont déterminants dans l'identification des sources de minéralisation d'une eau souterraine (Jain et al., 2005). Par ailleurs, une nappe d'eau confinée, c'est-àdire séparée de la surface par une formation géologique imperméable, sera moins exposée à la dilution des minéraux qu'elle contient puisqu'elle est moins en contact avec des eaux récentes provenant des précipitations. De même, une eau circulant lentement à travers les formations géologiques aura le temps de se charger en minéraux qu'une eau circulant rapidement (Gourcy et al., 2011). En résumé, la minéralisation finale d'une eau souterraine est fonction de la nature de la roche qui compose l'aquifère, du type de circulation des eaux au sein de la masse rocheuse, du temps de contact avec les minéraux, des phénomènes d'oxydo-réduction et du renouvellement de l'eau dans le réservoir par les apports météoriques.

L'hydrogéochimie est un outil
intéressant pour discriminer des eaux

d'origines superficielles ou profondes, récentes ou anciennes, exposées à la pollution ou non exposées. Les paramètres physicochimiques classiques utilisés sont: le $\mathrm{pH}$, la température, la conductivité électrique, la teneur en oxygène dissous et la concentration en éléments majeurs (Gourcy et al., 2011). Dans les départements de Daloa et Zoukougbeu, situés au Centre-Ouest de la Côte d'Ivoire, les eaux souterraines constituent les principales sources d'alimentation en eau pour les populations majoritairement rurales. Les caractéristiques physico-chimiques de ces eaux ont été abordées dans certains travaux : Ligban et al., 2009, Adjiri et al., 2018. Toutefois, les caractéristiques hydrogéochimiques restent peu connues. C'est dans ce contexte précis que s'inscrit la présente étude qui vise à indiquer à partir des caractéristiques physicochimiques des eaux souterraines, l'origine de la minéralisation de ces eaux. En d'autres termes, il est question dans cette étude de rechercher à partir des propriétés physicochimiques des eaux souterraines (puits et sources) des deux départements, l'origine de la minéralisation grâce aux simulations PHREEQC (PH, Redox, EQuilibrium, Code) et diagramme.

\section{MATÉRIEL ET MÉTHODES \\ Données géographiques et urbaines}

Situés au Centre-Ouest de la Côte d'Ivoire, les départements de Daloa et Zoukougbeu sont à environ $141 \mathrm{~km}$ de Yamoussoukro, la capitale politique et à environ $383 \mathrm{~km}$ d'Abidjan, la capitale économique. Ils couvrent une superficie d'environ $5400 \mathrm{~km}^{2}$ et sont situés entre la latitude $6^{\circ}$ et $7^{\circ}$ Nord et la longitude $7^{\circ}$ et $8^{\circ}$ Ouest (Figure 1). Ces départements comptent huit sous-préfectures : Daloa, Zoukougbeu, 
Domangbeu, Zahibo, Gboguhé, Bédiala, Gonaté et Gadouan. Ils sont limités par les départements de Vavoua et de Zuénoula au nord, les départements de Bangolo et Duékoué à l'ouest, les départements de Bouaflé et de Sinfra à l'est et le département d'Issia au sud.

\section{Régime climatique}

Le climat de la région est de type tropical à quatre saisons : la grande saison des pluies d'avril à mi-juillet, marquée par des orages, la petite saison sèche de mi-juillet à mi-septembre, la petite saison des pluies de mi-septembre à novembre et la grande saison sèche de décembre à mars. Ce climat conférait aux départements une végétation homogène constituée de forêt dense et humide au sud et de savane arborée au nord. Annuellement, la température moyenne est de 25,6 ${ }^{\circ} \mathrm{C}$ (Adjiri et al., 2018). Les saisons sèches et humides alternent avec des températures variant de $24,65{ }^{\circ} \mathrm{C}$ à $27,75^{\circ} \mathrm{C}$ en moyenne. Les pluies annuelles sont passées de 1868,5 mm en 1968 à 1120,4 mm en 2005, soit une baisse de $40 \%$ (Ligban et al., 2009). La dégradation de cette forêt est accélérée par l'intensification des cultures de rente (cacao, café, palmier à huile et hévéa). Les pratiques culturales extensives et itinérantes ainsi que l'exploitation non contrôlée des essences forestières ont fait reculer les limites de cette forêt. Cette situation perturbe gravement le climat de cette localité avec une incidence sur les ressources en eau mobilisables (Die, 2006).

Les formations qui constituent le substratum de la région ont subi une altération et latérisation caractérisant les régions à climat chaud et humide. Du point de vue lithologique, le socle est constitué par des granitoïdes de composition minéralogique constante. Il s'agit de granites alcalins à subalcalins contenus dans les formations métamorphiques (gneiss et migmatites) de composition très voisine. Ces formations sont très rarement visibles à l'affleurement. Elles sont masquées par une épaisse couverture d'altération argilo-sableuse. Le sol est donc constitué sur $20 \mathrm{~m}$ d'épaisseur environ de produits d'altérations des roches. Le relief de ces deux départements est constitué de plateau de 200 à $400 \mathrm{~m}$ d'altitude, coupé en maints endroits par des plaines et basfonds (Adjiri et al., 2018). En fait, il s'agit d'une pénéplaine monotone légèrement inclinée vers le sud. Elle regroupe un ensemble de dômes cristallins de faible altitude (300 à $400 \mathrm{~m}$ ) situé au nord et les bas plateaux du sud (200 à $300 \mathrm{~m}$ ). Les forages dans ces départements captent les eaux au niveau du socle cristallin fissuré et ne peuvent fournir qu'un débit maximum de $10 \mathrm{~m}^{3} / \mathrm{h}$ pour des profondeurs supérieures à $50 \mathrm{~m}$. Ce qui complique l'utilisation de forages pour l'alimentation en eau des grands centres urbains (Die, 2006).

\section{Techniques de prélèvement et d'analyses des échantillons}

Nous avons effectué deux campagnes d'échantillonnage en mars 2018. La première campagne a eu lieu dans la ville de Daloa et la seconde sur les axes Gonaté-DaloaZoukougbeu et Daloa-Vavoua-Gboguhé. Elles ont permis de prélever 14 échantillons de sols et 17 échantillons d'eaux (11 d'eaux de sources naturelles et 6 de puits traditionnels), dans 12 localités différentes (Figure 2), soit au moins un échantillon par localité avec un accent particulier dans la ville de Daloa. Les échantillons de sols ont été prélevés aux mêmes points que les échantillons d'eau et conditionnés dans des sachets alimentaires. L'écart entre le nombre des deux types d'échantillons se justifie par le fait qu'au niveau du département de Daloa, les aménagements réalisés autour de certains sites d'eaux de source ont rendu impossible le prélèvement des sols de proximité. Les analyses chimiques ont porté sur un échantillon de sol moyen. Elles ont été faites au Laboratoire de Chimie du Centre de Recherches Océanologiques (CRO) de Côte d'Ivoire. Les paramètres dosés sont : le nitrite, le nitrate, l'ammonium et le $\mathrm{pH}$.

Pour les échantillons d'eau, la démarche suivante a été respectée au cours de l'échantillonnage: Les flacons (1 L) ont été rincés 3 fois avec l'eau à analyser, puis remplis jusqu'à ras bord et fermés hermétiquement. Ils ont été ensuite conservés dans une glacière à une température basse d'environ $4{ }^{\circ} \mathrm{C}$. Les analyses ont été réalisées au bout de 48 heures après la prise d'échantillons et ont porté sur les ions les plus 
courants et les plus abondants dans les eaux souterraines. Il s'agit de quatre cations : $\mathrm{Ca}^{2+}$, $\mathrm{Mg}^{2+}, \mathrm{Na}^{+}, \mathrm{K}^{+}$et de quatre anions : $\mathrm{Cl}^{-}, \mathrm{HCO}_{3}^{-}$ , $\mathrm{SO}_{4}{ }^{2-}, \mathrm{NO}_{3}{ }^{-}$. Les chlorures $\left(\mathrm{Cl}^{-}\right)$ont été dosés par colorimétrie au thiocyanate de mercure, les sulfates $\left(\mathrm{SO}_{4}{ }^{2-}\right)$ par la méthode colorimétrique au sulfaver 4 et les nitrates $\left(\mathrm{NO}_{3}{ }^{-}\right)$par la méthode de réduction au cadmium (Méthode $\mathrm{HACH}$ et ce, sur spectromètre DR 6000). Les teneurs en bicarbonates ont été déterminées en mesurant le titre alcalimétrique complet (TAC) à l'aide d'un titrimètre digital de type $\mathrm{HACH}$, puisque le titre alcalimétrique est nul quand le $\mathrm{pH}$ est inférieur à 8,3. Le dosage s'est donc fondé sur la neutralisation d'un volume d'eau $(100 \mathrm{~mL})$ par un acide minéral en utilisant le vert méthyl rouge de bromocrésol comme indicateur coloré. La détermination des ions $\mathrm{HCO}_{3}{ }^{-} \mathrm{s}$ 'est faite à l'aide de la relation :

$\mathrm{HCO}_{3}{ }^{-}(\mathrm{mg} / \mathrm{L})=1,219 \times \mathrm{TAC}$, Avec TAC : taux d'alcalinité totale en $\mathrm{mg} / \mathrm{L}$ de $\mathrm{CaCO}_{3}$.

Pour la mesure de la turbidité, l'échantillon a été introduit dans un flacon de mesure normalisé et mesuré dans un turbidimètre. Quant aux cations, ils ont été dosés par spectrophotométrie d'émission atomique (ICP-AES) dont la source est du plasma généré par couplage inductif conformément à la norme ISO-11885.

Auparavant, sur le terrain, la température, le $\mathrm{pH}$, la conductivité électrique (CE) et le TDS (Total Dissolved Solids) ont été mesurés à l'aide d'un multimètre de marque HACH HQ 40d.

\section{Détermination de l'origine de la minéralisation des eaux}

Pour la détermination de l'origine de la minéralisation, les logiciels géochimiques PHREEQC interactive 3.4.0-12927 et Diagramme 5.0 ont été utilisés pour le calcul des indices de saturation par rapport aux principaux minéraux carbonatés et évaporites. Selon Tessier (2012), le logiciel PHREEQC est un modèle géochimique qui fonctionne en considérant le système à l'équilibre thermodynamique. Les calculs réalisés par le logiciel sont basés sur l'équilibre chimique entre tous les éléments présents dans le système étudié. Dans la présente étude, cet équilibre chimique a été assuré entre l'eau et la roche encaissante et a permis d'apprécier l'origine de la minéralisation des eaux. Les résultats ont été vérifiés avec ceux du logiciel Diagramme 5.0 qui est également un logiciel géochimique comportant un module permettant d'effectuer les mêmes calculs. Les paramètres tels que la pression partielle de $\mathrm{CO}_{2}\left(\mathrm{pCO}_{2}\right)$ et les indices de saturation (IS) des eaux générés après la simulation, permettent de prédire la réaction minéralogique pouvant contribuer à la saturation des eaux souterraines. En effet, l'indice de saturation (IS) d'un minéral est donné par l'équation :

IS $=\log \left(\mathrm{I}_{\mathrm{AP}} / \mathrm{K}_{\mathrm{SP}}\right)$, où $\mathrm{I}_{\mathrm{AP}}$ est le produit de l'activité ionique de la solution et $\mathrm{K}_{\mathrm{SP}}$, le produit de solubilité à un moment et une température donnés.

Un IS inférieur à zéro indique que l'eau souterraine est sous-saturée par rapport à ce minéral. Une telle valeur reflète le caractère d'une eau ayant une faible quantité de minéraux et provenant d'une formation avec laquelle le temps de séjour a été relativement court. Un indice IS supérieur à zéro indique une eau souterraine sursaturée par rapport à la matière minérale et reflète les eaux d'un aquifère relativement bien concentrées en matière minérale et ayant passé un temps de séjour relativement long dans l'aquifère. Cependant, la sursaturation peut également être produite par d'autres facteurs tels que la dissolution incongruante, l'effet d'ion commun, l'évaporation, l'augmentation rapide de la température et du $\mathrm{CO}_{2}$ en solution (Ako et al., 2010).

\section{RÉSULTATS}

\section{Les analyses réalisées sur les échantillons de sols}

Les résultats des analyses chimiques réalisées sur les échantillons de sols sont présentés dans le Tableau 1.

Les sols des différentes localités sont acides. La moyenne des $\mathrm{pH}$ est de 4,94. Ils varient entre un minimum de 3,86 à Dagbaboua et un maximum de 5,97 à Gonaté. On note également que les teneurs en nitrate dans les sols sont plus élevées que celles des autres paramètres mesurés. Elles varient entre un minimum de 13,9 ppm à Sapia et un maximum de 224,50 ppm à Dagbaboua. Ces 
teneurs présentent certes des avantages pour les sols culturaux, mais sont dangereuses pour les eaux de proximités (eaux de surfaces et nappes superficielles).

\section{Les mesures in situ}

Le Tableau 2 présente les résultats des mesures réalisées in situ au niveau des sources et puits. Les différentes valeurs de paramètres mesurés sont inférieures aux valeurs guides de l'OMS (2011). Le rapport moyen TDS/CE est de 0,45. Ce rapport est généralement de 0,5 pour les solutions ioniques fortes et de 0,7 pour les solutions ioniques faibles. Dans les départements de Daloa et Zoukougbeu, la conductivité des eaux de source et de puits oscille entre 33,7 et $218,9 \mu \mathrm{S} / \mathrm{cm}$. Ces eaux se situent donc entre les eaux faiblement minéralisées $\quad(0<\mathrm{CE}<80)$ et les eaux relativement bien minéralisées $(300<\mathrm{CE}<500)$. Le rapport TDS/CE devrait donc être proche de 0,7. Cependant, il est plutôt proche de 0,5; qui est le rapport correspondant aux solutions fortement minéralisées. Cela révèle l'existence d'ions mineurs non dosés. On note également une forte fluctuation autour des moyennes des paramètres TDS, conductivité et turbidité. Les écarts autour des moyennes de TDS et CE seraient liés à la nature lithologique de certains aquifères qui généraient accidentellement des eaux relativement bien minéralisées. Au niveau de la turbidité, les écarts seraient également liés, non seulement à la nature lithologique de certaines formations traversées au cours du passage de l'eau jusqu'à la résurgence, mais aussi à la profondeur et le niveau de salubrité de l'environnement immédiat de certaines sources et puits. D'ailleurs, la plupart des eaux sont relativement chaudes $\left(26,2^{\circ} \mathrm{C}\right.$ $\left.<\mathrm{T}<29,3^{\circ} \mathrm{C}\right)$ et acides $(4,2<\mathrm{pH}<5,91)$. Ces fortes températures enregistrées pourraient s'expliquer par le fait que ces sources seraient issues de nappes peu profondes.

\section{Les analyses chimiques réalisées au laboratoire}

Le résultat des analyses effectuées au laboratoire sur les échantillons d'eau est présenté dans le Tableau 3. Les teneurs en ions majeurs dosés sont toutes inférieures aux valeurs guides OMS (2011) recommandées pour les eaux de consommation humaine. Toutefois, on note des teneurs relativement élevées d'ions nitrates, supérieures à la dizaine d'unités dans les eaux de sources $(38,07 \mathrm{mg} / \mathrm{L})$ et de puits $(14,61 \mathrm{mg} / \mathrm{L}) \mathrm{de}$ Zoupangbeu ainsi que dans le puits de Toroguhé $(14,17 \mathrm{mg} / \mathrm{L})$. Ces teneurs sont certes inférieures aux valeurs guides OMS (50 $\mathrm{mg} / \mathrm{L}$ ), mais restent tout de même préoccupantes $\mathrm{du}$ fait de leur origine anthropique.

\section{Interactions eau-roche}

Les indices de saturation (IS) par rapport à la calcite, l'aragonite, la dolomite, au gypse, l'anhydrite, l'halite et la sylvite et les pressions partielles de $\mathrm{CO}_{2}$ simulés sont présentés dans le Tableau 4. On constate que la plupart des indices de saturation des différents minéraux sont négatifs à l'exception de ceux des sources de Gbokora et Toroguhé et du puits de Zoupangbeu légèrement saturés respectivement au gypse et à l'anhydrite. Les indices négatifs indiqueraient une soussaturation à la plupart des minéraux indiqués. Ce facteur serait dû probablement au temps de séjour relativement court des eaux dans l'encaissant. Les indices positifs quant à eux, traduiraient une légère sursaturation aux minéraux concernés. Cette formation de minéraux (dépôts) expliquerait les teneurs très faibles à relativement faibles des ions constitutifs des minéraux indiqués. Aussi, on note l'absence de certains minéraux dans certaines eaux. Par exemple, au niveau des eaux de source, la calcite, l'aragonite, la dolomite et l'anhydrite sont absents à Gbokora et le gypse à Toroguhé. Au niveau des eaux de puits, la dolomite, le gypse et l'anhydrite sont absents dans les eaux de Zakoua et le gypse dans les eaux de Zoupangbeu. D'autre part, les pressions partielles de $\mathrm{CO}_{2}\left(\mathrm{pCO}_{2}\right)$ varient de $10^{\circ}$ (source de Toroguhé et puits de Zoukangbeu) à $10^{1,07} \mathrm{~atm}$ (puits de Zakoua). Ces valeurs, plus élevées que celle de l'atmosphère $\left(10^{-3,5}\right.$ $\mathrm{atm})$, suggèrent que le système est fermé au $\mathrm{CO}_{2}$ de l'atmosphère et ouvert au $\mathrm{CO}_{2}$ du sol. L'origine du $\mathrm{CO}_{2}$ peut être principalement attribuée à la remontée mantélique liée à la respiration des racines et/ou à la 
décomposition de la matière organique du sol. Cependant, les teneurs en acide carbonique sont relativement faibles et nulles par endroit. Elles sont nulles au niveau de la source de Toroguhé et des eaux de puits de Zakoua et Zépréguhé. Ces faibles valeurs suggèrent de faibles dégagements de $\mathrm{CO}_{2}$. Cela se justifie par le fait qu'il y a moins de possibilités pour que le système racinaire des végétaux et la matière organique de surface arrivent à de grande profondeur par rapport aux formations géologiques en présence (migmatites avec des intrusions granitiques (Figure 3)). Les teneurs en nitrates plus élevées dans les eaux de sources (teneur $\max =38,07 ;$ moyenne $=8,91$ $\mathrm{mg} / \mathrm{L}$ ) que dans les eaux de puits (teneur $\max =14,61 ;$ moyenne $=6,82 \mathrm{mg} / \mathrm{L}$ ) justifieraient une contamination par les formations superficielles au cours de la résurgence des sources. Au niveau des puits, elles proviendraient soit des eaux parasites déversées dans le puits au cours de l'exploitation, soit de la nitratation du $\mathrm{NH}_{4}{ }^{+}$ contenu dans le feldspath altéré. Le premier facteur justifie l'origine anthropique du nitrate.

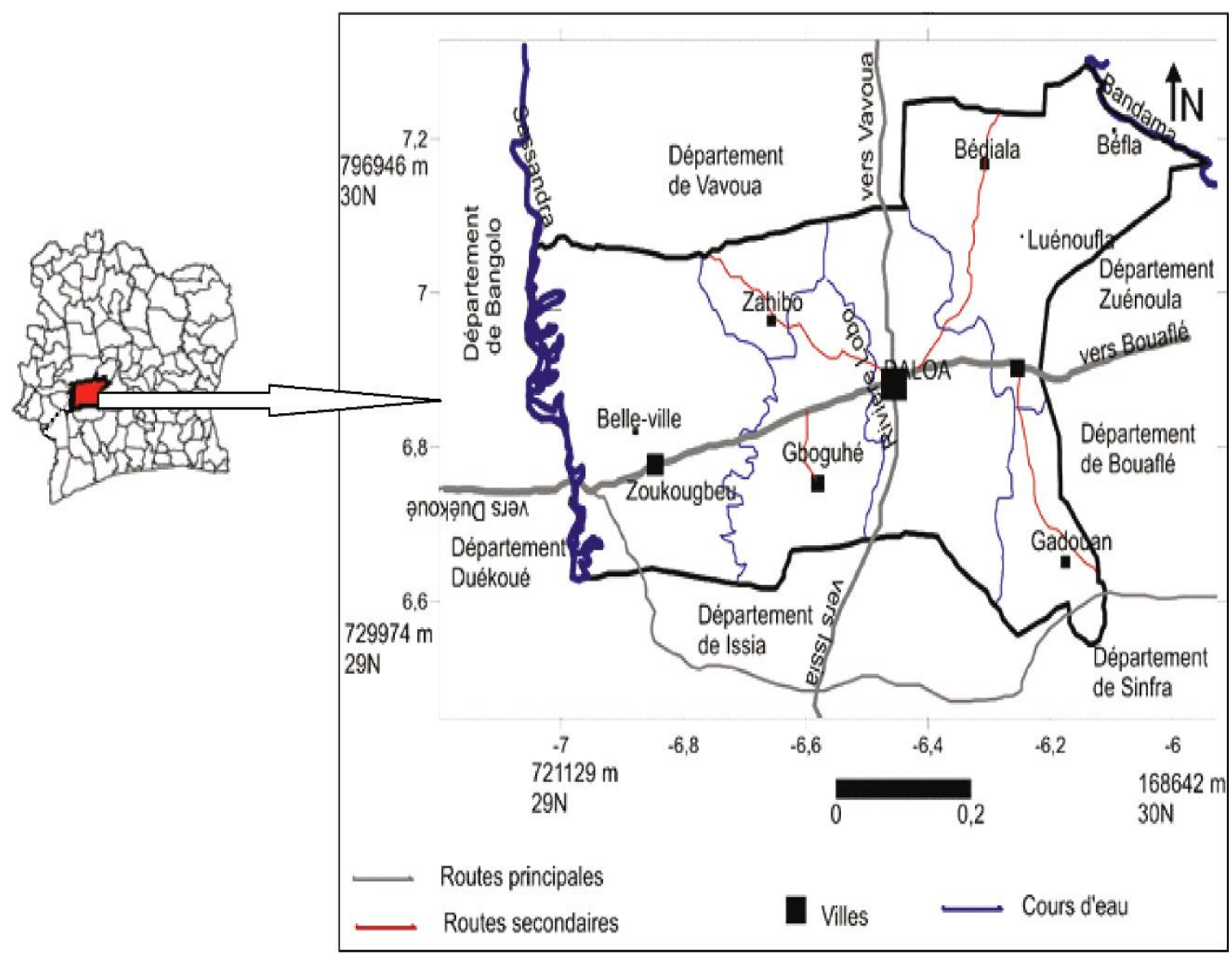

Figure 1 : Localisation de la zone d'étude. 


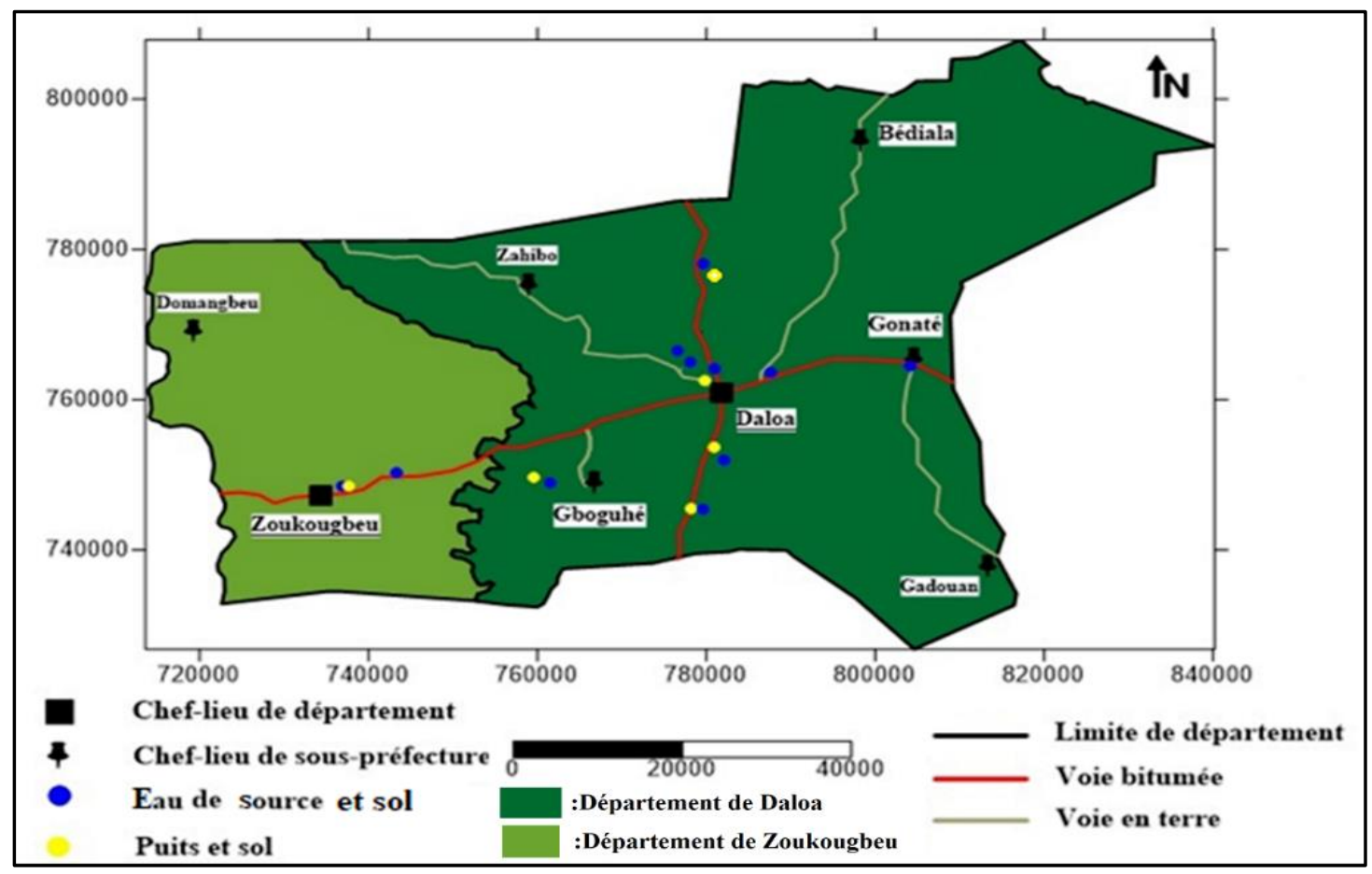

Figure 2 : Carte des stations de prélèvement des échantillons.

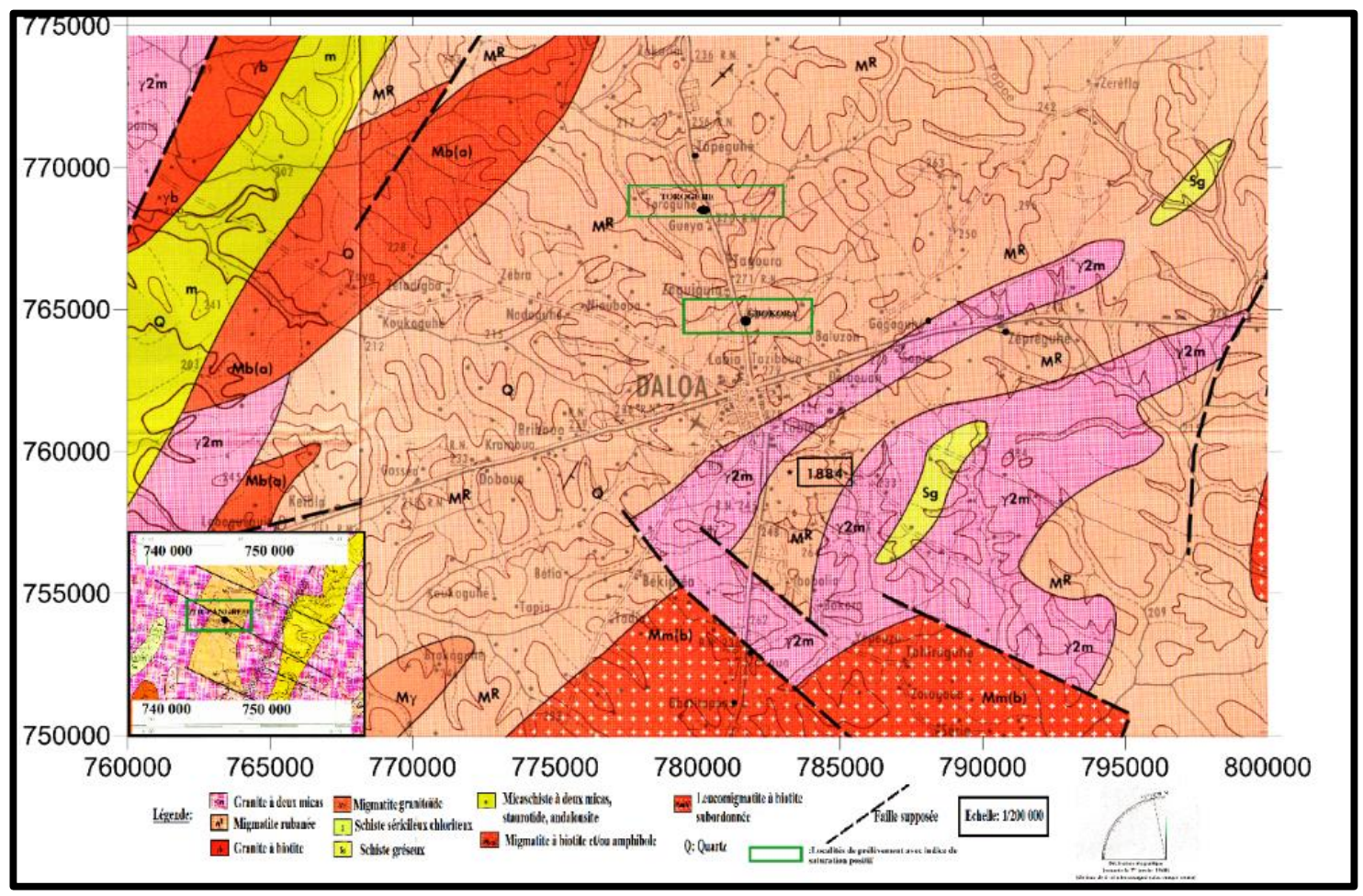

Figure 3 : Géologie de la zone d'étude (Ahimon et al., 1990).

Tableau 1 : Résultat des analyses réalisées sur les échantillons de sols. 


\begin{tabular}{lcccc}
\hline \multicolumn{1}{c}{ Localités } & $\left.\mathbf{N O}_{\mathbf{2}^{-}} \mathbf{~ p p m}\right)$ & $\mathbf{N H}_{\mathbf{4}}{ }^{+}(\mathbf{p p m})$ & $\mathbf{N O}_{\mathbf{3}^{-}} \mathbf{( \mathbf { p p m } )}$ & $\mathbf{p H}$ \\
\hline Zoupangbeu* & $<\mathrm{LD}$ & 0,48 & 84 & 4,73 \\
Toroguhé & 0,02 & 0,56 & 15,2 & 5,18 \\
Zoukoubgeu source * & 0,31 & 0,57 & 100 & 5,11 \\
Gbokora & 0,29 & 0,38 & 69 & 5,02 \\
Gonaté & 0,4 & 0,95 & 151 & 5,97 \\
Gbetitapéa & 0,26 & 0,49 & 81 & 4,34 \\
Zoupangbeu Carrefour* & 0,62 & 0,89 & 62 & 5,49 \\
Sapia & 0,02 & 0,41 & 13,9 & 5,56 \\
Gogoguhé & 0,3 & 0,68 & 78 & 5,23 \\
Zoukougbeu puit* & 0,01 & 0,12 & 110 & 4,4 \\
Zakoua & 0,35 & 0,24 & 111 & 4,12 \\
Zépréguhé & 0,02 & 0,33 & 117 & 4,17 \\
Tapeguhé & 0,06 & 0,27 & 88 & 5,92 \\
Dabagboua & 0,17 & 1,12 & 224,50 & 3,86 \\
\hline \multicolumn{1}{c}{ Minimum } & $<\mathbf{L D}$ & $\mathbf{0 , 1 2}$ & $\mathbf{1 3 , 9}$ & $\mathbf{3 , 8 6}$ \\
\multicolumn{1}{c}{ Maximum } & $\mathbf{0 , 6 2}$ & $\mathbf{1 , 1 2}$ & $\mathbf{2 2 4 , 5 0}$ & $\mathbf{5 , 9 7}$ \\
$\quad$ Moyenne & $\mathbf{0 , 2 0}$ & $\mathbf{0 , 5 4}$ & $\mathbf{9 3 , 1 9}$ & $\mathbf{4 , 9 4}$ \\
\hline
\end{tabular}

* : échantillons prélevés dans le département de Zoukougbeu ; LD : limite de détection.

Tableau 2 : Résultat des mesures réalisées in situ.

\begin{tabular}{|c|c|c|c|c|c|c|}
\hline Localités & TDS (mg/L) & $\mathbf{T}\left({ }^{\circ} \mathbf{C}\right)$ & pH & $\mathrm{CE}(\boldsymbol{\mu} \mathrm{S} / \mathrm{cm})$ & Turbidité (UTN) & TDS/CE \\
\hline \multicolumn{7}{|c|}{ EAUX DE SOURCE } \\
\hline Zoupangbeu* & 100,20 & 26,2 & 4,62 & 218,9 & 23,50 & 0,46 \\
\hline Toroguhé & 47,60 & 27,3 & 4,95 & 106,5 & 1,24 & 0,45 \\
\hline Zoukoubgeu* & 17,13 & 27,5 & 4,55 & 37,70 & 64,10 & 0,45 \\
\hline Gbokora & 26,30 & 29,1 & 5,17 & 56,0 & 23,30 & 0,47 \\
\hline Etat-major & 53,60 & 29,3 & 5,91 & 113,5 & 3,70 & 0,47 \\
\hline Gonaté & 37,70 & 29,2 & 4,38 & 87,3 & 4,92 & 0,43 \\
\hline Gbetitapéa & 15,33 & 26,3 & 4,49 & 33,7 & 2,19 & 0,45 \\
\hline Zoupangbeu & & & & & & 0,45 \\
\hline Carrefour* & 21,13 & 26,4 & 4,90 & 46,5 & 146,00 & \\
\hline Sapia & 33,70 & 28,6 & 4,38 & 76,9 & 48,20 & 0,44 \\
\hline Gogoguhé & 31,80 & 26,4 & 4,75 & 69,7 & 90,70 & 0,46 \\
\hline Minimum & 15,33 & 26,2 & 4,38 & 33,7 & 1,24 & $\mathbf{0 , 4 3}$ \\
\hline Maximum & 100,20 & 29,3 & 5,91 & 218,9 & 146,00 & 0,47 \\
\hline Moyenne & 38,45 & 27,6 & 4,81 & 84,7 & 40,78 & 0,45 \\
\hline Ecart-type & 25,01 & 1,3 & 0,46 & 54,5 & 47,70 & $\mathbf{0 , 0 1}$ \\
\hline \multicolumn{7}{|c|}{ EAUX DE PUITS } \\
\hline Artisanat & 39,40 & 28,3 & 5,06 & 83,9 & 8,60 & 0,47 \\
\hline
\end{tabular}


O. A. ADJIRI et al. / Int. J. Biol. Chem. Sci. 13(4): 2388-2401, 2019

\begin{tabular}{|c|c|c|c|c|c|c|}
\hline Zoukougbeu* & 25,00 & 28,2 & 5,00 & 57,6 & 63,40 & 0,43 \\
\hline Zoupangbeu* & 43,20 & 28,3 & 4,47 & 97,7 & 30,90 & 0,44 \\
\hline Zakoua & 34,60 & 27,8 & 4,90 & 77,7 & 67,80 & 0,45 \\
\hline Zépréguhé & 35,10 & 26,8 & 5,12 & 77,7 & 17,00 & 0,45 \\
\hline Tapeguhé & 48,80 & 28,7 & 5,14 & 111,2 & 1,37 & 0,44 \\
\hline Dabagboua & 30,20 & 27,2 & 4,02 & 67,2 & 1,51 & 0,45 \\
\hline Minimum & 25,00 & 26,8 & 4,02 & 57,6 & 1,37 & 0,43 \\
\hline Maximum & 48,80 & 28,7 & 5,14 & 111,2 & 67,80 & 0,47 \\
\hline Moyenne & 36,61 & 27,9 & 4,81 & 81,8 & 27,22 & 0,45 \\
\hline Ecart-type & $\mathbf{7 , 9 8}$ & $\mathbf{0 , 7}$ & $\mathbf{0 , 4 2}$ & 18,0 & 28,13 & $\mathbf{0 , 0 1}$ \\
\hline \multicolumn{7}{|l|}{ Valeur guide } \\
\hline OMS & & $22-25$ & $6,5-9,5$ & $1000-1400$ & $<5$ & \\
\hline
\end{tabular}

CE : Conductivité Electrique ; * : échantillons prélevés dans le département de Zoukougbeu.

Tableau 3 : Résultat des analyses chimiques réalisées au laboratoire.

\begin{tabular}{|c|c|c|c|c|c|c|c|c|}
\hline Localités & $\begin{array}{l}\mathrm{Ca}^{2+} \\
(\mathrm{mg} / \mathrm{L})\end{array}$ & $\begin{array}{c}\mathrm{Mg}^{2+} \\
(\mathrm{mg} / \mathrm{L})\end{array}$ & $\begin{array}{c}\mathrm{Na}^{+} \\
(\mathrm{mg} / \mathrm{L})\end{array}$ & $\begin{array}{c}\mathbf{K}^{+} \\
(\mathrm{mg} / \mathrm{L}) \\
\end{array}$ & $\begin{array}{l}\mathrm{HCO}_{3}^{-} \\
(\mathrm{mg} / \mathrm{l})\end{array}$ & $\begin{array}{c}\mathrm{Cl}^{-} \\
(\mathrm{mg} / \mathrm{L})\end{array}$ & $\begin{array}{c}\mathrm{SO}_{4}^{-2} \\
(\mathrm{mg} / \mathrm{L})\end{array}$ & $\begin{array}{l}\mathrm{NO}_{3}^{-} \\
(\mathrm{mg} / \mathrm{L})\end{array}$ \\
\hline \multicolumn{9}{|c|}{ EAUX DE SOURCE } \\
\hline Zoupangbeu** & 9,2 & 3,17 & 8,84 & 2,72 & 5,49 & 16 & 2 & 38,07 \\
\hline Toroguhé & 4,8 & 1,47 & 4,92 & 1,51 & 13,42 & 8,9 & 1 & 14,17 \\
\hline Zoukoubgeu** & 1,6 & 0,24 & 2,60 & 0,80 & 5,98 & 4,7 & 9 & 5,31 \\
\hline Gbokora & 0,5 & 0,54 & 2,66 & 1,04 & 6,10 & 6,0 & 5 & 0,90 \\
\hline Etat-major & 2,81 & 2,72 & 12,50 & 3,31 & 34,16 & 9,0 & 1 & 0,50 \\
\hline Gonaté & 1,2 & 0,73 & 5,25 & 1,62 & 6,10 & 9,5 & 1 & 7,97 \\
\hline $\begin{array}{l}\text { Gbetitapéa } \\
\text { Zoupangbeu }\end{array}$ & 0,8 & 0,24 & 2,43 & 0,75 & 7,32 & 4,4 & 4 & 1,77 \\
\hline Carrefour** & 1,2 & 0,98 & 3,54 & 1,09 & 36,6 & 6,4 & 11 & 6,64 \\
\hline Sapia & 1,2 & 0,49 & 5,47 & 1,68 & 6,83 & 9,9 & 6 & 6,20 \\
\hline Gogoguhé & 0,8 & 0,24 & 5,36 & 1,65 & 5,12 & 9,7 & 14 & 7,53 \\
\hline Minimum & 0,5 & 0,24 & 2,43 & 0,75 & 5,12 & 4,4 & 1 & $\mathbf{0 , 5 0}$ \\
\hline Maximum & 9,2 & 3,17 & 12,50 & 3,31 & 36,60 & 16,0 & 14 & 38,07 \\
\hline Moyenne & 2,41 & $\mathbf{1 , 0 8}$ & 5,36 & 1,62 & 12,71 & 8,45 & 5,3 & 8,91 \\
\hline \multicolumn{9}{|c|}{ EAUX DE PUITS } \\
\hline Artisanat & 0,41 & 0,50 & 6,97 & 6,66 & 2,44 & 9,0 & 5 & 1,70 \\
\hline Zoukougbeu** & 3,6 & 1,71 & 1,33 & 0,41 & 9,03 & 2,4 & 7 & 8,41 \\
\hline Zoupangbeu** & 0,4 & 0,49 & 5,25 & 1,62 & 3,66 & 9,5 & 1 & 14,61 \\
\hline Zakoua & 0,4 & 0,10 & 4,03 & 1,24 & 2,44 & 7,3 & 9 & 7,08 \\
\hline Zépréguhé & 2,8 & 0,49 & 3,92 & 1,21 & 17,08 & 7,1 & 5 & 6,20 \\
\hline Tapéguhé & 5,6 & 2,93 & 7,46 & 2,30 & 19,89 & 13,5 & 3 & 4,43 \\
\hline Dabagboua & 0,8 & 0,49 & 5,47 & 1,68 & 0,73 & 9,9 & 8 & 5,31 \\
\hline Minimum & 0,4 & 0,10 & 1,33 & 0,41 & $\mathbf{0 , 7 3}$ & 2,4 & 1 & 1,70 \\
\hline Maximum & 5,6 & 2,93 & 7,46 & 6,67 & 19,89 & 13,5 & 9 & 14,61 \\
\hline
\end{tabular}




\begin{tabular}{lcccccccc} 
Moyenne & $\mathbf{2 , 0 0}$ & $\mathbf{0 , 9 5}$ & $\mathbf{4 , 9 2}$ & $\mathbf{2 , 1 6}$ & $\mathbf{7 , 8 9}$ & $\mathbf{8 , 4}$ & $\mathbf{5 , 4}$ & $\mathbf{6 , 8 2}$ \\
\hline $\begin{array}{l}\text { Valeurs guides } \\
\text { OMS* }\end{array}$ & $\mathbf{7 0}$ & $\mathbf{5 0}$ & $\mathbf{2 0 0}$ & $\mathbf{1 2}$ & $\mathbf{2 0 0}$ & $\mathbf{2 5 0}$ & $\mathbf{2 5 0}$ & $\mathbf{5 0}$ \\
\hline * : valeurs guides pour les eaux de consommation humaine (OMS, 2011$).$ \\
** : échantillons prélevés dans le département de Zoukougbeu.
\end{tabular}

Tableau 4 : Indice de saturation des eaux et pression partielle du $\mathrm{CO}_{2}\left(\mathrm{pCO}_{2}\right)$.

\section{SOURCES}

\begin{tabular}{|c|c|c|c|c|c|c|c|c|c|}
\hline Localités & Calcite & Aragonite & Dolomite & Gypse & Anhydrite & halite & sylvite & $\begin{array}{l}\mathrm{pCO}_{2} \\
\text { éq.[atm] }\end{array}$ & $\begin{array}{l}\mathrm{H}_{2} \mathrm{CO}_{3} \\
\text { éq. }[\mathrm{mmol} / \mathrm{L}]\end{array}$ \\
\hline Zoupangbeu* & $-4,99$ & $-5,13$ & $-10,62$ & $-3,88$ & $-4,10$ & $-6,88$ & $-6,96$ & 0,14 & 4,60 \\
\hline $\begin{array}{l}\text { Zoupangbeu } \\
\text { carrefour }\end{array}$ & $-4,75$ & $-4,89$ & $-9,76$ & -4 & $-4,21$ & $-7,66$ & $-7,73$ & 0,49 & 16,20 \\
\hline Zoukoubgeu & $-5,73$ & $-5,87$ & $-12,44$ & $-3,93$ & $-4,14$ & $-7,92$ & $-8,00$ & 0,18 & 5,90 \\
\hline Toroguhé & $-4,51$ & $-4,66$ & $-9,71$ & - & 0,16 & $-7,37$ & $-7,46$ & 0 & 0 \\
\hline Gbokora & - & - & & 1,42 & 0 & $-7,8$ & $-7,79$ & 0,64 & \\
\hline Gonaté & $-5,99$ & $-6,13$ & $-12,33$ & $-5,01$ & $-5,21$ & $-7,31$ & $-7,40$ & 0,28 & 8,74 \\
\hline Gbetitapea & $-6,01$ & $-6,15$ & $-12,72$ & $-4,57$ & $-4,78$ & $-7,97$ & $-8,05$ & 0,25 & 8,44 \\
\hline Sapia & $-5,95$ & $-6,09$ & $-12,44$ & $-4,24$ & $-4,44$ & $-7,66$ & $-7,73$ & 0,32 & 9,83 \\
\hline Gogoguhé & $-5,92$ & $-6,06$ & $-12,54$ & $-4,06$ & $-4,27$ & $-7,27$ & $-7,35$ & 0,10 & 3,21 \\
\hline \multicolumn{10}{|c|}{ PUITS } \\
\hline Artisanat & $-6,19$ & $-6,33$ & $-12,44$ & $-4,78$ & $-4,99$ & $-7,21$ & $-6,80$ & 0,02 & 0,74 \\
\hline Zoukougbeu & $-4,74$ & $-4,88$ & $-9,96$ & $-3,70$ & $-3,91$ & $-8,51$ & $-8,59$ & 0,10 & 3,13 \\
\hline Zoupangbeu & $-6,61$ & $-6,75$ & $-13,28$ & - & 0,14 & $-7,31$ & $-7,40$ & 0 & 0 \\
\hline Zakoua & $-6,36$ & $-6,5$ & - & 0 & 0 & $-7,54$ & $-7,62$ & 1,07 & 0 \\
\hline Zépréguhé & $-4,47$ & $-4,62$ & $-9,88$ & $-3,95$ & $-4,17$ & $-7,56$ & $-7,64$ & 0,14 & 4,55 \\
\hline Tapeguhé & $-4,08$ & $-4,22$ & $-8,58$ & $-3,90$ & $-4,11$ & $-7,01$ & $-7,10$ & 0,16 & 4,92 \\
\hline Dabagboua & $-7,48$ & $-7,62$ & $-15,33$ & $-4,29$ & $-4,5$ & $-7,28$ & $-7,36$ & 0,07 & 2,44 \\
\hline
\end{tabular}

\section{DISCUSSION}

Les départements de Daloa et Zoukougbeu sont situés dans le Centre-Ouest de la Côte d'Ivoire, précisément dans le domaine protérozoïque. Les formations géologiques identifiées au niveau de la plupart des sites de prélèvement sont les migmatites avec des intrusions granitiques. Les résultats des analyses physico-chimiques, révèlent pour les paramètres mesurés in situ $(\mathrm{pH}$, 
température, conductivité électrique et TDS), des valeurs inférieures aux valeurs guides de l'OMS (2011) pour l'eau de boisson. Les TDS mesurés sont tous inférieurs à $600 \mathrm{mg} / \mathrm{L}$. Mouncherou et al. (2011) considèrent pour ces valeurs de TDS, des eaux suffisamment diluées pour être potables. Le pH moyen est de 4,81. Ces eaux sont donc acides et ce caractère acide pourrait s'expliquer par le fait qu'elles seraient issues de nappes d'altérites. En effet, plusieurs auteurs (Ligban et al., 2009 ; Ahoussi et al., 2013 ; et Adjiri et al., 2018) ont montré dans leurs différents travaux que les eaux de puits et de sources de cette région sont issues de nappes peu profondes. Pour ces auteurs, l'acidification de ces eaux serait due à la disparition des minéraux primaires facilement altérables, à la nondissociation de l'acide organique et de l'acide carbonique qui jouent un rôle de tampon pour limiter l'acidité de l'eau et à la forte transpiration de la forêt qui accroît la diminution du pH. Cependant, Ligban et al. (2009) attribuent cette acidité à l'abondance de quartz comme l'en témoignent les types de formations identifiés (des migmatites avec des intrusions granitiques). Cette abondance de quartz confère donc aux roches une résistance au phénomène d'hydrolyse responsable de la minéralisation des eaux souterraines et expliquerait les faibles valeurs de TDS et de $\mathrm{CE}$ obtenues. Plusieurs travaux réalisés en Côte d'Ivoire et en Afrique révèlent ce caractère acide et la faible minéralisation des eaux en zone de socle. En Côte d'Ivoire, il s'agit des travaux de Goné et al. (2004) dans la région du Sud-Est, d'Adiaffi (2008) dans la zone de contact "socle-bassin sédimentaire" du Sud-Est, de Ligban et al. (2009) dans le degré carré de Daloa. En Afrique, ce sont les travaux de Mouncherou et al. (2011) dans la région du Bamoun au Cameroun.

Au niveau chimique, le sodium $\left(\mathrm{Na}^{+}\right)$, le potassium $\left(\mathrm{K}^{+}\right)$et le calcium $\left(\mathrm{Ca}^{2+}\right)$, constituants triviaux des migmatites, constituent la majorité des cations avec des teneurs relativement élevées en $\mathrm{Na}^{+}$. Ce dernier proviendrait des formations riches en minéraux ferromagnésiens sodiques, tel que l'albite. Ces teneurs relativement élevées ont été indiquées par Ligban et al. (2009) dans les eaux de puits du degré carré de Daloa. Ahoussi et al. (2011 et 2013), Yao et al. (2012), Aka et al. (2013) dans leurs différents travaux réalisés respectivement à Kossihouen dans le District d'Abidjan, à MangouinYrongouin dans la sous-préfecture de Biankouman, dans la région du Sud-Ouest de la Côte d'Ivoire et à Abengourou, ont également noté des teneurs relativement moins élevées de $\mathrm{Na}^{+}$. Cependant, certains auteurs africains comme Gnazou et al. (2015) au Togo, Mfonka et al. (2015) au Bénin et Tamungang et al. (2016) au Cameroun ont plutôt mis en évidence une dominance du $\mathrm{Ca}^{2+}$ dans les eaux en zone de socle. Le $\mathrm{Ca}^{2+}$ fait partie de la structure des minéraux comme le plagioclase. Sa libération, nécessite l'hydrolyse acide des plagioclases. La minéralisation calcique des eaux est donc sous l'influence de l'altération et de l'hydrolyse des feldspaths dans ces pays. Au contraire, le cation alcalin $\mathrm{K}^{+}$est très peu présent dans les eaux des départements de Daloa et Zoukougbeu, avec une moyenne de 1,74 $\mathrm{mg} / \mathrm{L}$. Ces résultats sont comparables à ceux des auteurs indiqués précédemment ayant réalisé des travaux en zone de socle ivoirien. Aussi, en Afrique, ils sont comparables à ceux de Mfonka et al. (2015) et Gnazou et al. (2015), ayant réalisé leurs travaux en zone de socle respectivement au Bénin et au Togo. Concernant les sels nutritifs, le nitrate constitue l'un des composés azotés dominants dans les eaux souterraines des deux départements. Rodier et al. (2009) ont montré que les valeurs en nitrates des eaux naturelles oscillent généralement entre 0 et $15 \mathrm{mg} / \mathrm{L}$. La source de Zoupangbeu avec la concentration maximale de $38,07 \mathrm{mg} / \mathrm{L}$ enregistre une valeur supérieure à quelques dizaines d'unité. Toutefois, cette teneur indique que ces eaux sont de bonne qualité car elle reste inférieure à la valeur guide de $50 \mathrm{mg} / \mathrm{L}$ proposée par 
l'OMS (2011) mais avec détection d'une contamination d'origine anthropique. Cette teneur serait due au lessivage des sols suivi de la nitratation du $\mathrm{NH}_{4}^{+}$contenu dans les feldspaths et/ou à une contamination anthropique suite à l'urbanisation (ce point d'eau est situé en contre bas des lieux d'habitation) ou à l'utilisation des engrais ou selon Yao (2009) et Yao al. (2012) à la décomposition de la matière organique. D'autre part, les résultats de modélisation PHREEQC et diagramme ont montré que les indices de saturation des principaux minéraux carbonatés tels que la calcite, l'aragonite et la dolomite et des principales évaporites telles que l'halite et la sylvite sont négatives. Dans un système fermé à l'atmosphère, ces indices négatifs supposent une moindre dissolution des minéraux des roches pour saturer les eaux. Ces résultats sont en accord avec ceux de Djemba et al. (2015) en Algérie. Les travaux de ces derniers ont indiqué une soussaturation des eaux souterraines par rapport aux principaux minéraux évaporitiques et carbonatés. Oga et al. (2009) dans la région de Tiassalé (Côte d'Ivoire) ont également trouvé une sous-saturation des eaux par rapport à la calcite et la dolomite.

\section{Conclusion}

Deux campagnes d'échantillonnages des eaux souterraines ont été menées dans les départements de Daloa et Zoukougbeu dans le but d'identifier à partir d'étude hydrogéochimique, l'origine de la minéralisation des eaux. Les résultats des analyses physico-chimiques et des modélisations PHREEQC et diagramme révèlent des eaux acides, faiblement minéralisées et sous-saturées aux différents minéraux carbonatés et évaporitiques. Le système est fermé à l'atmosphère. Les seuls agents responsables donc de la minéralisation des eaux sont la dissolution des minéraux des roches encaissantes et le $\mathrm{CO}_{2}$ issus de la remontée mantélique liée à la respiration des racines et/ou à la décomposition des matières organiques du sol. La faible minéralisation et la sous-saturation traduiraient un niveau de renouvellement élevé et un âge récent des eaux souterraines.

\section{CONFLITS D'INTÉRÊTS}

Les auteurs déclarent ne pas avoir d'intérêts concurrents.

\section{CONTRIBUTIONS DES AUTEURS}

OAA est l'investigateur principal de ce manuscrit. NA a contribué aux prélèvements des échantillons. ID a contribué à l'analyse des données et résultats d'analyses ainsi qu'à leur interprétation et discussion. BK a participé à la lecture et la correction du manuscrit. BD a assuré la supervision générale des travaux.

\section{RÉFÉRENCES}

Adiaffi B. 2008. Apport de la géochimie isotopique, de l'hydrochimie et de la télédétection à la connaissance des aquifères de la zone de contact "soclebassin sédimentaire" du sud-est de la Côte d'Ivoire. Thèse de doctorat unique en sciences de la terre, Université Paris Sud, Orsay, p. 230.

Adjiri OA, Aka N, Soro TD, Afessi AC, Konaté D, Soro N. 2018. Caractérisation des ressources en eaux alternatives de la ville de Daloa : impacts sur la santé et implications dans le développement régional. astee-tsm.fr., 12: 89-114.

Ahoussi KE, Koffi YB, Kouassi AM, Soro GM, Soro N, Biémi J. 2013. Étude hydrochimique et microbiologique des eaux de source de l'Ouest montagneux de la Côte d'Ivoire : Cas du village de Mangouin-Yrongouin (sous-préfecture de Biankouman). J. Appl. Biosci., 63: 4703-4719.

Ahoussi KE, Oga YMS, Yao KB, Kouassi AM, Nagnin S, Biémi J. 2011. Caractérisation hydrogéochimique et 
microbiologique des ressources en eau $\mathrm{du}$ site d'un Centre d'Enfouissement Technique (CET) de Côte d'Ivoire : cas du CET de Kossihouen dans le District d'Abidjan (Côte d'Ivoire). Int. J. Biol. Chem. Sci., 5(5): 2114-2132. DOI: http://dx.doi.org/10.4314/ijbcs.v5i5.32.

Aka N, Bamba SB, Soro G, Soro N. 2013. Étude hydrochimique et microbiologique des nappes d'altérites sous climat tropical humide : cas du département d'Abengourou (Sud-Est de la Côte d'Ivoire). Larhyss Journal, 16: 31-52.

Boubakar HA. 2011. Aquifères superficiels et profonds et pollution urbaine en Afrique : Cas de la communauté urbaine de Niamey (NIGER). Thèse de doctorat, Université Abdou Moumouni de Niamey, Niger, p. 249.

De Marsily G et Besbes M. 2017. Les eaux souterraines. Responsabilité \& environnement, $86: 25-30$.

Die KK. 2006. Renforcement de l'alimentation en eau potable de la ville de Daloa à partir du barrage de Buyo en Côte d'Ivoire. Mémoire de fin de formation de l'EIER-ETSHER, Ouagadougou, p. 77

Djenba S, Boudoukha A, Bencer S. 2015. Approche hydrochimique de l'aquifère superficiel de Ain Djacer, Est algérien. Larhyss Journal, 24: 281-299.

Gnazou MDT, Assogba K, Sabi BE, Bawa LM. 2015. Qualité physico-chimique et bactériologique des eaux utilisées dans les écoles de la préfecture de Zio (Togo). Int. J. Biol. Chem. Sci., 9(1): 504-516. DOI http://dx.doi.org/10.4314/ijbcs.v9i1.43.

Goné DL, Savané I, Goblé MM. 2004. Caractéristiques physico-chimiques majeures des eaux des aquifères fissurées de la région d'Agboville (Sud-Ouest de la côte d'ivoire). Rev. ivoir. Sci. Technol., 5: 117-133.

Gourcy L et Petelet-Giraud E. 2011. Utilisation des outils isotopiques pour la délimitation des aires d'alimentation des captages destinés à l'alimentation en eau potable. Rapport BRGM/RP-59729-FR, Orleans, p. 62.

Jain P, Sharma JD, Sohu D, Sharma P. 2005. Chemical analysis of drinking water of villages of Sanganer Tehsil, Jaipur District. Int. J. Environ. Sci. Dev., 2(4) : 373-379.

Ligban R. 2013. Caractérisation hydrogéologique et origine des eaux de sources naturelles dans le degré carré de Daloa (Centre-Ouest ; Côte d'Ivoire). Thèse de doctorat unique, Université Nangui Abrogoua, Abidjan, p.135.

Ligban R, Gone DL, Kamagate B, Saley MB, Biemi J. 2009. Processus hydrogéochimiques et origine des sources naturelles dans le degré carré de Daloa. Int. J. Biol. Chem. Sci., 3(1): 3847.

Mfonka Z, Ndam NJR, Ndjigui PD, Zammouri M, Kpoumie A, Rasolomanana E. 2015. Hydrochimie et potabilité des eaux du bassin versant du Nchi dans le plateau Bamoun (Ouest Cameroun). Int. J. Biol. Chem. Sci., 9(4): 2200-2218.

DOI: http://dx.doi.org/10.4314/ijbcs.v9i4.39

Mohan R, Singh AK, Tripathi JK, Chowdhary GC. 2000. Hydrochemistry and quality assessment of groundwater in Naini industrial area, District Allahabad, Uttar Pradesh. Jour. Geol. Soc. India, 55(1): 77-89.

Mouncherou FO, Moundi A, Ngoupayou NRJ, Ako AA, Wandji P. 2011. Paramètres chimiques et source lithologique de la minéralisation des eaux souterraines des aquifères du Plateau Bamoun, Ligne Volcanique du Cameroun (LVC). Review of the bulgarian geological society, 72 (part 13): 67-78.

Oga MS, Lasm T, Yao TK, Soro N, Saley MB, Kouassi D, Gnamba F. 2009. 
Caractérisation chimique des eaux des aquifères de fracture: Cas de la région de Tiassalé en Côte d'Ivoire, European Journal of Scientific Research, 31(1): 72-87.

OMS (Organisation Mondiale de la Santé). 2011. Guidelines for Drinking-water Quality, Recommendations (4th edn). WHO Library Cataloguing-inPublication Data: Switzerland.

Rajmohan N, Elango L, Ramachandran S, Natarajan M. 2003. Major ion correlation in groundwater of Kancheepuram region, South India. Indian J. Environ. Health, 45(1): 5-10.

Rodier J, Legube B, Merlet N, Brunet R. 2009. L'Analyse de l'Eau (9ème edn). Dunod: Paris.

Tamungang NEB, Nchofua FB, Mofor NA, Djoko YT. 2016. Contrôle de la qualité des eaux domestiques dans le village Babessi au Nord-Ouest Cameroun. Int. J. Biol. Chem. Sci., 10(3) : 1382-1402. DOI:

http://dx.doi.org/10.4314/ijbcs.v10i3.37
Tessier E. 2012. Diagnostic de la contamination sédimentaire par les métaux/métalloïdes dans la rade de Toulon et mécanismes contrôlant leur mobilité. Thèse de doctorat, Université du Sud Toulon Var, Toulon, p. 269.

Yao KT, Oga M-S, Fouché O, Baka D, Pernelle C, Biémi J. 2012. Évaluation de la potabilité chimique des eaux souterraines dans un bassin versant tropical : cas du Sud-Ouest de la Côte d'Ivoire. Int. J. Biol. Chem. Sci., 6(6): 7069-7086. DOI: 10.4314/ijbcs.v6i6.42

Yao KT. 2009. Hydrodynamisme dans les aquifères de socle cristallin et cristallophyllien du sud-ouest de la côte d'ivoire : cas du département de Soubré. Apports de la télédétection, de la géomorphologie et de l'hydrogéochimie. Thèse de Doctorat unique, Université de Cocody, Abidjan, p. 285. 